\title{
Tablet allergen immunotherapy (TAIT) requires tight management
}

\author{
Giorgio Ciprandi
}

Received: 26 October 2020 / Accepted: 21 November 2020 / Published online: 11 January 2021

C) Springer Medizin Verlag GmbH, ein Teil von Springer Nature 2021

Keywords Placebo · Allergic rhinitis - Symptoms · Perception · Improvement

\section{To the editor,}

A very recent, large trial showed that the 300IR house dust mite (HDM) tablet allergen immunotherapy (TAIT) was effective and safe for patients with HDMdriven allergic rhinitis [1]. The outcomes of this randomized controlled trial seem to be exciting, as suggesting fruitful practical information.

However, the patients allocated to the placebo arm experienced an impressive reduction (by about 40\%) of the primary outcome, the combined score (CS) for the symptoms' severity and rescue medications use over time. Indeed, such an large reduction is truly outstanding to be ascribable to a simple placebo effect. Notably, placebo patients had a lower CS at baseline. The authors speculated that the placebo group's clinical benefits might have been due to a series of factors, including the improved care, the so-called Hawthorne effect, and the patients' expectations about the TAIT effectiveness. However, improved care was probably the most relevant factor. Instead, a similar study showed that the placebo treatment reduced the same primary outcome by about $30 \%$ after one year [2]. This discrepancy could depend on the severity of allergic rhinitis (AR) symptoms at enrollment. The patients included in the previous trial suffered from more intense symptoms than patients in the current study. There is an agreement that the AIT treatment effect is higher in moderate to severe symptomatic patients

G. Ciprandi, MD $(\bowtie)$

Casa di Cura Villa Montallegro, Via P. Boselli 5, 16146 Genoa, Italy

gio.cip@libero.it
[3]. Accordingly, a patient with the mild disease is not a candidate for AIT.

Another relevant topic was the high compliance to the AIT schedule (above 90\%), suggesting that the patients had been intensely cared for. The study design included ten visits in just one year. In common practice, AR patients usually have a specialized visit once or twice per year at most [4]. This point is, therefore, crucial for the best standard of care. Frequent and thorough visits guarantee an optimal acceptance of the AIT by the patient. This fact is determinant in achieving maximal adherence and awareness of receiving optimal care. Consequently, the patients perceive to improve in their allergy.

The authors also emphasized the proportion of patients who withdrew from the study during the first few months of treatment, but in real-life the dropout rate due to safety would have been handled differently, with patient's exact instructions on how to manage adverse events with oral antihistamines, and when to interrupt and restart AIT.

This study's lesson suggests that patient engagement is crucial in managing allergic patients, mainly concerning the AIT strategy. Allergists should be convincing and willing to explain and discuss the rationale, mechanisms of action, correct work-up, duration, safety, costs, and long-lasting benefits of AIT [5]. Moreover, the allergist should help the patient to choose the administration route and may need to adapt the dose by prescribing to the patient several days with the 100 IR dose to target optimal tolerance and effectiveness.

In conclusion, the ideal candidate for AIT should have moderate-severe symptoms. AIT management requires continuous contact with the patients, possibly also through telemedicine. This trial demonstrated that regular and timely follow-up ensured very high adherence to the TAIT schedule. This issue is 
crucial as discontinuation is frequent in patients selfadministering AIT. On the other hand, COVID-19 allows using telemedicine to adequately follow allergic patients to guarantee optimal control of allergies [6]. Moreover, tablets, which are handy and easy, could be a reliable option for all patients. However, TAIT requires close management.

Conflict of interest G. Ciprandi declares that he has no competing interests.

\section{References}

1. Demoly P, Corren J, Creticos P, De Blay F, Gevaert P, Hellings P, et al. A $300^{\circ} \mathrm{IR}$ sublingual tablet is an effective, safe treatment house-dust-mite-induced allergic rhinitis: an international, double-blind, placebo-controlled, randomized Phase III clinical trial. J Allergy Clin Immunol. 2020. (In press).
2. Demoly P, Emminger W, Rehm D, Backer V, Tommerup L, Kleine-Trebbe J. Effective treatment of house dust miteinduced allergic rhinitis with 2 doses of the SQ HDM SLIT-tablet: results from a randomized, double-blind, placebo-controlled phase III trial. J Allergy Clin Immunol. 2016;137:444-51.

3. Howarth P, Malling H-J, Molimard M, Deviller P. Analysis of allergen immunotherapy studies shows increased clinical efficacy in highly symptomatic patients. Allergy. 2012;67:321-7.

4. Ciprandi G, Incorvaia C, Tosca MA. The behavior of Italian allergists in prescribing allergen immunotherapy for house dust mites allergy. Immunotherapy. 2018;10:1343-8.

5. Ciprandi G, Natoli V, Puccinelli P, Incorvaia C. Allergic rhinitis: the eligible candidate to mite immunotherapy in the real world. Allergy Asthma Clin Immunol. 2017;13:11.

6. KlimekL,PfaarO, WormW,Bergmann KC,BieberT, BuhlR, et al. Allergen immunotherapy in the COVID 19 pandemic: An ARIA-EAACI-AeDA-GPA-DGAKI position paper. Allergo J. 2020;29(3):17-25. 